\title{
Integrated Design of a Motion Cueing Algorithm and Motion-Base Mechanism for a Wright Flyer Simulator
}

By

Sunjoo Advani

Director Simulation \& Training, ADSE B.V., Hoofddorp, The Netherlands

SunjooA@ADSE.NL
Ruud Hosman

Director, Aerospace Man-Machine Systems Consulting, Delft, The Netherlands

R.Hosman@AMSConsult.Demon.NL

\author{
Nils Haeck \\ Mathematics and Motion Control \\ Specialist, Hellevoetsluis, The \\ Netherlands \\ N.Haeck@Chello.NL
}

\begin{abstract}
Given a specific flight vehicle and a flying task - in this case the 1903 Wright Flyer - a forward design process can be utilized to specify a corresponding flight simulator motion cueing system. This process, presented in this paper, is based on the analysis of the pilot-vehicle control loop by using a pilot model incorporating both visual and vestibular feedback, and the aircraft dynamics. After substituting the model for the simulated aircraft, the analysis tools are used to adjust the washout filter parameters with the goal of restoring pilot control behaviour. This process allows the objective specification of the motion cueing algorithm. Then, based on flight files representative for the operational flight envelope, the required motion system space is determined. The motion-base geometry is established based on practical limitations, as well as criteria for the stability of the platform with respect to singular conditions. With this process the characteristics of the aircraft, the tasks to be simulated, and the missions themselves are taken into account in defining the simulator motion cueing system.
\end{abstract}

\section{I ntroduction}

Orville Wright, the pilot of the first powered airplane, was faced with an exceptionally difficult task - to manually control an unstable flight vehicle with no prior formal training in powered flight. He was purely reliant on the capacity to sense the vehicle motions, to visualize its flight path, and to adjust his control outputs to maintain a stable pilot-vehicle system. It was an unstable airplane, particularly in its pitch motions as both Orville and Wilbur quickly discovered, and required very careful manipulation of the "forward rudder" (as their canard was then referred to). Correction for the turbulence present at

Copyright (C) 2002 The American Institute of Aeronautics and Astronautics Inc. All rights reserved.
Kill Devil Hill that day made the task indeed a very challenging one ${ }^{12}$.

Since the first flights of the Wrights on 17 December 1903, both aerospace and simulation technologies have progressed hand-in-hand. In the 1970's, simulators became equipped with motion cueing systems. When coupled with powerful visual display systems, the modern simulator can provide a powerful sensation of selfmotion. However, even though today's civil aviation regulations require the presence of motion cueing systems on high-end training simulators, there are yet no cueing criteria for motion cueing systems.

The choice of motion system characteristics is based on the requirements of the simulator user and, if applicable, regulatory requirements. The major part of the motion system requirements, in particular the motion space, system bandwidth, maximum load, etc. are actually dependent upon the operation required to be simulated. The current lack of motion cueing criteria, however, is the primary reason that there has been no real progress in the development of motion cueing algorithm and the systematic design of simulator motion systems during the last decades. As a result, the final specifications are primarily based on experience, rather than the actual need.

Teunissen ${ }^{17}$ suggests that for the selection of the training means from CBT to FFS, it is necessary to first specify the training objective (the level of proficiency that the trainee has to master), and the training need (the difference between the objective, and the proficiency before the training). If the selection of the FFS is based on such a specification, then the motion system characteristics must still be defined. It is known that the flight maneuvers to be simulated, and the properties of the motion cueing algorithm, determine the required motion space of the motion system.

In an effort to overcome this status quo, Advani and Hosman $^{4}$ presented in 2000 a scheme for an integrated design of the motion cueing algorithm and the motion system, Fig. 1. Based on this strategy, the specification of 
both the motion cueing algorithm (also known as the washout filter) and the motion system geometry can be specified if the required knowledge, information and software are available.

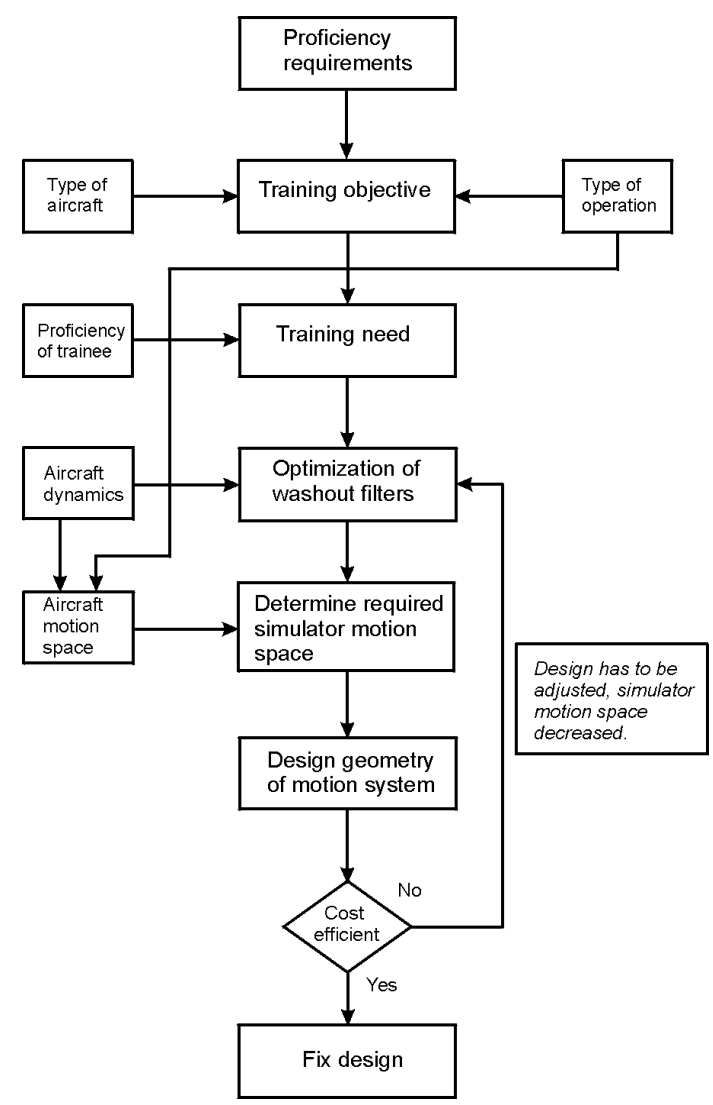

Figure 1 - Integrated motion cueing system design process 4,11

Since 2000, several customers have shown interest in the integrated design method. Numerous projects to design these washout filters, the motion system geometry ${ }^{2}$ and the combined integrated design of the motion system and washout algorithm ${ }^{11}$ have been performed since (Ref. 2), or are currently in progress.

The present paper will discuss the details, merits and shortcomings of this design procedure, based on an example employing the 1903 Wright Flyer.

First an overview of the process and its building blocks will be presented. Thereafter, the results are shown and discussed and conclusions drawn.

\section{Simulator Motion Cueing System Design Process and Elements}

The authors have developed a systematic means of specifying simulator motion cueing systems, comprised of the motion drive algorithm and the motion-base mechanism. The design process follows four essential steps:

1. Adjust a validated pilot model to the given dynamics of the aircraft.

2. Introduce simulator parameters (time delays and the washout filter form), and adjust the washout algorithm parameters in order to restore the pilotsimulator system behavior as closely as possible to the pilot-aircraft behaviour.

3. Based on the stabilized pilot-simulated aircraft system, generate simulator trajectories representing characteristic maneuvers.

4. Design a simulator motion-base that contains these trajectories as best as possible.

The integrated design is based on a sequential analysis, which leads to the optimization of the motion cueing algorithm and the geometry of the motion system. For the analysis, the following models are required:

1. An aerodynamic model of the aircraft.

2. A model describing a pilot's skill-based control behaviour incorporating the visual and vestibular feedback.

3. A model describing a pilot's motion perception incorporating visual and vestibular stimulation.

4. A model describing the general form of the motion cueing algorithm.

5. A set of (simulated) flight files covering the operation to be simulated.

7. A tool to optimize the design of the motion system, including a means of comparing the actual available workspace with that required by the above simulations.

In the Wright Flyer example discussed in this paper, linear models will be used. This is not a requisite, but for the analysis based on non-linear models, more detailed information and knowledge would be required, and the analysis itself is much more extensive. Both the aircraft model and the flight files were provided from the University of Liverpool. The flight trajectory files were generated during a generic piloted moving-base simulation of the Wright Flyer.

As described by Hosman ${ }^{9}$, a distinction must be made regarding the influence of motion feedback at each of the three different levels of behaviour as described by Rasmussen ${ }^{14}$. These levels are skill-based (manual control), rule-based and knowledge-based behaviour.

\section{Pilot-model adaptation to the aircraft-model}

The first step required for this analysis is to adapt the pilot model to the aircraft dynamics in the attitude control loop, Fig. 2. 


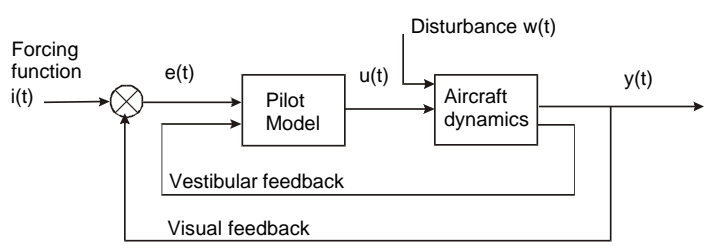

Figure 2 - The closed loop with the pilot and the aircraft and the visual and vestibular feedback loops

The pilot model used in this type of analysis must be able to process the influence of the visual and vestibular feedback ${ }^{7,8,10}$ in the inner attitude control loop on the pilot's skill-based manual control behaviour. Based on extensive research on the influence of visual and vestibular perception of motion stimuli, and on motion perception and control behaviour in tracking tasks, a descriptive pilot model has been developed and validated ${ }^{8,10}$. The aim was to attain a model capable of describing the influence of the visual and vestibular stimulation induced by the aircraft motions on a pilot's control behaviour. The final model is depicted in Fig. 3.

With the experiments described by Hosman ${ }^{8,10}$, the dynamics of the sensors and the interaction between the visual and the vestibular systems were evaluated. In this model, the human motion sensors - each of which is described by a transfer function - are placed in parallel and convert the stimuli (the attitude, angular rate, and angular acceleration) to the sensory outputs $R_{i}(\omega)$. The differences in sensor dynamics are due to the fundamental differences between the visual and the vestibular system: The visual system is position and rate sensitive, while the vestibular system is sensitive to angular accelerations and specific forces.

In the perception and decision process, the many sensory outputs have to be integrated into one single output. Based on the experimental results during tracking tasks, the model output is generated by a weighted sum of the sensory outputs. Each individual weighting factor $W_{i}$ emphasizes the contribution of each sensory output.

Next, the time delay resulting from the information processing and a simulation of the total neuromuscularmanipulator control system are incorporated into the model.

Due to the high bandwidth character of a well-designed neuromotor-manipulator control system $\left(\omega_{n} \gg \omega_{c}\right)$, the information processing delay and the neuromotormanipulator system can be modelled by one single lumped time delay $\tau_{I}$. Taking into consideration the effective time delay of the human operator in tracking tasks ${ }^{13}$, and the characteristics of the visual system, Hosman ${ }^{8}$ initially set $\tau_{I}=0.2 \mathrm{~s}$ to simultaneously represent the information time delay and the neuromuscular dynamics. This value is valid for tracking tasks with a side stick as the manipulator, and system dynamics that allow a high closed-loop bandwidth $\left(\omega_{c}>3\right.$ $\mathrm{rad} / \mathrm{sec}$ ). For system dynamics corresponding with those of transport aircraft, a time delay and neuromuscular dynamics (that account for the influence of the aircraft control system) have to be incorporated within the model.

When a pilot adjusts his/her behaviour to a certain control task, the first objective is to achieve an acceptable level of tracking performance. Normally, this tracking performance can be quantified by the mean square of the tracking error. When the pilot would try to minimize the tracking error alone, his control actions would not be taking into account the aircraft characteristics, structural loads and passenger comfort, for example. Therefore, if the mean square error were the only component of the cost function used to adapt the model to the aircraft dynamics, this would drive the model parameters to a value for best performance regardless the effect on the

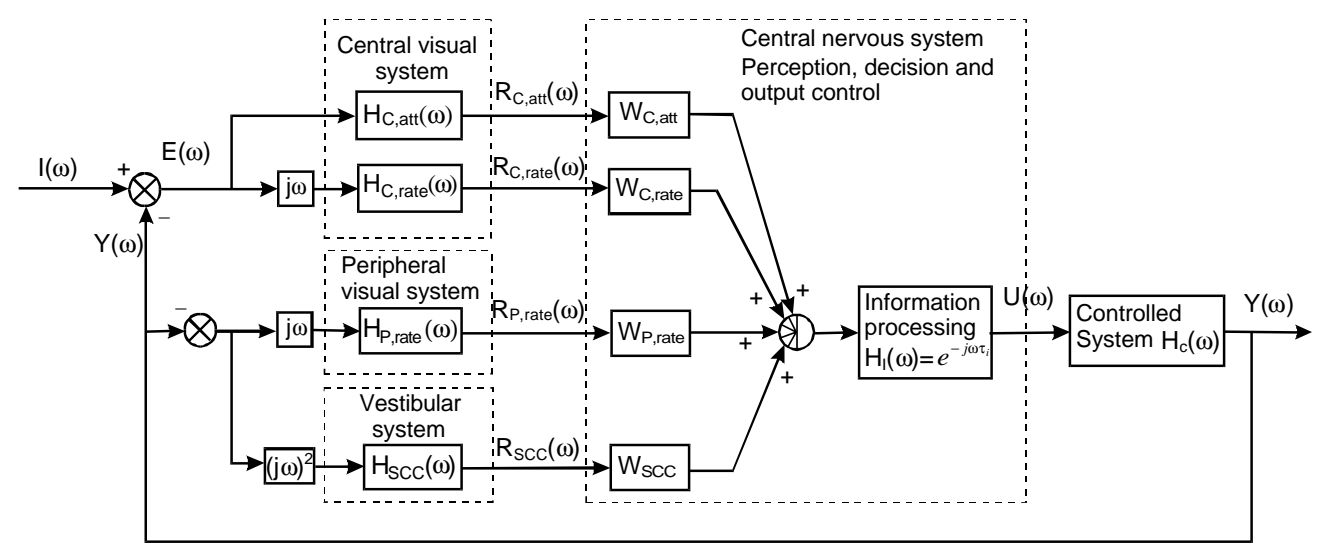

Figure 3 - Block diagram of the descriptive pilot model in the maneuver task 
dynamic characteristics of the closed loop. In reality, the pilot will normally consider putting more effort into the task as a function of the benefit of the resulting performance improvement, and relative to the corresponding increase in workload. For these reasons, to bring the workload effect into account, the mean square of the control signal $\delta$ and its derivative $\dot{\delta}$ have to be added to the cost function.

There is another consideration: When a pilot tries to improve tracking performance, he will also increase his gain. This will result in an increase of the crossover frequency $\omega_{c}$ and a decrease in phase margin $\varphi_{m}$. A gain that is too high will reduce the stability of the control loop. So, the choice of the cost function should aim at the following:

- Good tracking performance

- Effective control effort

- Adequate bandwidth and stability of the control loop as expressed in the crossover frequency and in the phase margin

In order to achieve these goals, the following cost function can be applied.

$$
J=\Sigma\left(e^{2}+Q \cdot \delta^{2}+R \cdot \dot{\delta}^{2}\right)
$$

Where $e$ is the tracking error, and $\delta$ is the control output. The weighing factors $\mathrm{Q}$ and $\mathrm{R}$ in the cost function depend primarily on the aircraft characteristics, and on the task to be performed, i.e. the disturbance or maneuver task.

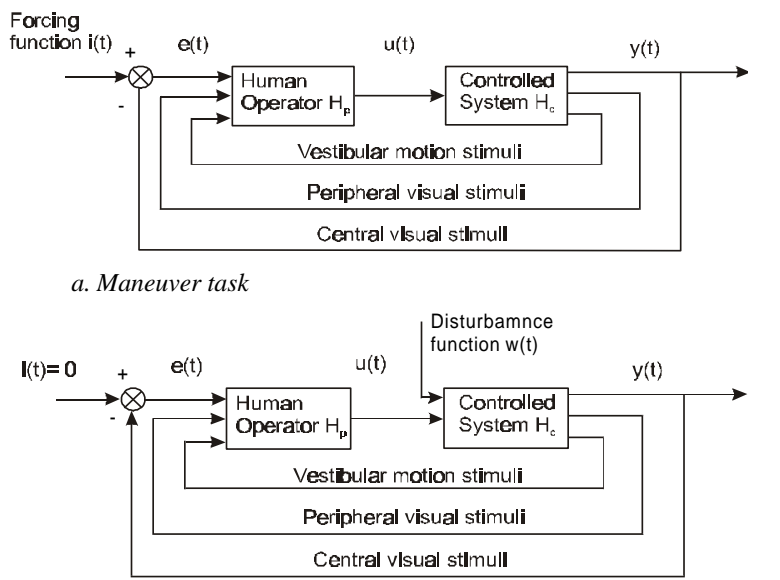

b. Disturbance task

Figure 4 - Differences in the influence of motion feedback in the maneuver task and the disturbance task

\section{Task Dependence}

With this model, the influence of visual and vestibular motion feedback in both the maneuver task and the disturbance task can be described. The advantage of the model is that by describing the influence of the visual and vestibular feedback on a pilot's control behaviour, the only free model parameters are the sensory weighting coefficients $W_{i}$, as shown in Fig. 3.

As shown in Fig. 4, there is an important difference in the feedback of the controlled system motion-stimuli between the maneuver task, and the disturbance task. This leads to a difference in the describing functions of the pilot model for both tasks.

If the pilot has to perform the control task with the central display and motion feedback, the model transfer function for the maneuver task based on the pilot model of Fig. 3 is given by Equation 2 .

$H_{p, \text { Man }}(\omega)=\frac{\left[W_{C . a t t} \cdot H_{C, a t t}(\omega)+W_{C, \text { rate }} \cdot H_{C, \text { rate }}(\omega) \cdot j \omega\right]}{\left[1+W_{S C C} \cdot H_{S C C}(\omega) \cdot(j \omega)^{2} \cdot K_{I} e^{-j \omega \tau_{I}} \cdot H_{c}(\omega)\right]} \cdot K_{I} e^{-j \omega \tau_{I}}$,

Conversely, the transfer function for the disturbance task:

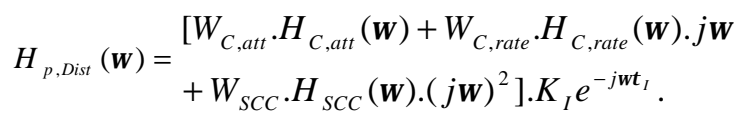

The cost function of Eq. 1 will be used to adjust the descriptive pilot model to the dynamics of the aircraft. To prevent the adjustment to a minimum of the cost function outside the normal operation area of the pilot, additional constrains may be required in order to attain a practical and sound result of the optimization.

\section{Motion Washout Parameter Adjustment}

After the pilot model has been adapted to the particular aircraft dynamics, the closed loop of Fig. 2 is adapted to the closed loop of the simulated aircraft, Fig. 5. In this scheme, three elements are added to the pilot-aircraft control loop: a lumped simulation time delay, the washout filter, and the motion system. In the present analysis, it is assumed that the motion system behaves perfectly in terms of its dynamics and has a gain of 1 .

Due to the changes of the control loop as caused by the simulation, the pilot will adapt his behaviour to the simulated-aircraft control loop.

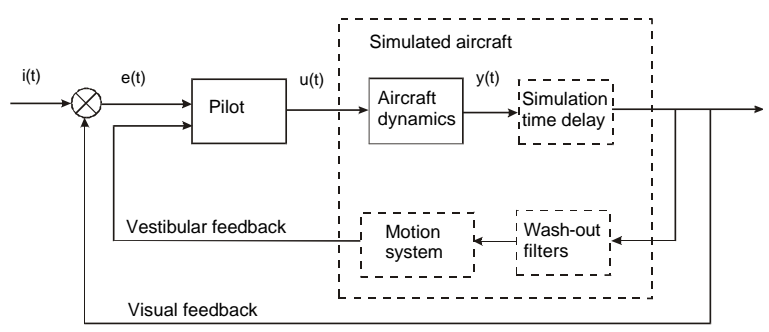

Figure 5 - The pilot simulated-aircraft control loop 
Using the pilot and aircraft model as the basis, the washout algorithm parameters are adjusted by hypothesizing that the cost function used to adapt the pilot model to the aircraft dynamics indeed describes the pilot's control strategy.

In the end, the motion washout algorithm coefficients remain the only parameters that may be adjusted in this phase. When the transfer function of these algorithms is introduced into the pilot-vehicle system, their coefficients can be adjusted then through a mathematical optimization. In the end, the optimization should try to restore the pilot control behaviour as closely as possible to the original behaviour. This is accomplished by using the same weighing factors in the cost function $\mathrm{J}$, see Eq. 1, in finding the optimal washout parameters. The resulting control strategy by the pilot can then be maintained in the simulator as it originally was in the aircraft.

For the adaptation of the washout filters to the pilot model and aircraft dynamics in the present project, the following arguments have been applied:

For a pilot's skill-based control behaviour, the inner attitude control loops are of direct importance ${ }^{9}$. Therefore, the simulation of the pitch angle $\theta$ and the roll angle $\varphi$ are of primary interest. When applying the classical washout filter, the high-pass rotational filter and the low-pass tilt-coordination filters generate the pitch and roll angles. The highfrequency components pass through the high-pass filter. The low-frequency components pass through the gravity component $g$ sine $\theta$ or $g$ sine $\varphi$ cosine $\theta$ of the specific force, and are filtered by the tiltcoordination filter. Therefore, the washout filter's influence on the simulated aircraft pitch attitude can be simplified as shown in Figure 6. For the

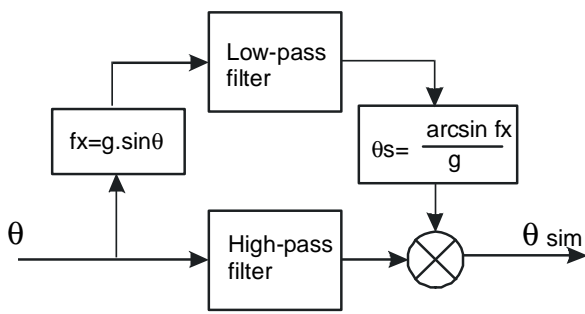

Figure 6 - Simplified scheme of the washout influence on the simulation of the pitch angle

asymmetric roll attitude, the lateral specific force is zero in a coordinated maneuver and the simulator roll angle results from the high pass rotation filter.

The remaining washout filter parameters, namely the high pass translational filters, have a direct influence on a pilot's rule-based and knowledge based behaviour. For these levels of behaviour, the perception of the aircraft motions in the environment directly impacts the performance. A general motion perception model is required in order to analyse and optimize the translational filters. Such a motion perception model is under development at TNO-Human Factors Institute in Soesterberg, the Netherlands. Bos et al ${ }^{5}$ applied this model to the motion perception during the take-off run of a transport aircraft. Although further development and evaluation is required, this model may be applied to the optimization of the translational filters in the washout algorithm. Therefore, in the present analysis the translational filter parameters have to be chosen based on the authors' experience.

In the end, this step generates the required motion drive algorithm parameters in order that the pilot perceives and responds to the simulated vehicle in a way that is as similar as possible to the vehicle.

Once the washout algorithm has been established, the required motion space can be determined.

\section{Required Simulator Motion Envelope}

In order to define objectively the six-degrees-of-freedom motion space that is required, measured state variables of an aircraft are passed through the motion drive algorithm in order to generate the "ideal" simulator trajectories. If the drive algorithm has been adjusted to the task and aircraft as reported earlier in this paper, and the maneuvers are measured under similar flight conditions with the same (or similar aircraft), then a simulator that reproduces these trajectories should generate a perception and control output by the pilot just like in the aircraft. This is the basis of the remainder of this strategy.

\section{Mechanism Design}

The performance capability of a motion-cueing system can be characterized by its kinematic envelope (or "workspace"), and its dynamic characteristics, such as phase delay and damping. The latter are a function of the mechanical power available to move the moving platform, its mass properties, and its bandwidth. The motion workspace is, however, a direct function of the architecture of the motion-cueing mechanism.

Modern flight simulator motion-bases generally utilise a mechanism known as the Stewart Platform ${ }^{16}$, or "hexapod", which was originally proposed in 1938 for the testing of vehicle tires. This mechanism is comprised of a base-frame, six actuator legs (the jacks), and an upper moving platform, which carries the payload. The legs are attached in pairs, via gimbal joints, to the upper and lower platforms near the vertices of their respective triangular frames (Fig. 7). 
The kinematic envelope (workspace) of a hexapod is limited and highly coupled. For example, a pitch-up attitude of 20 degrees (common during the simulation of a take-off) will reduce the available motions in all other directions and rotations to nearly zero.

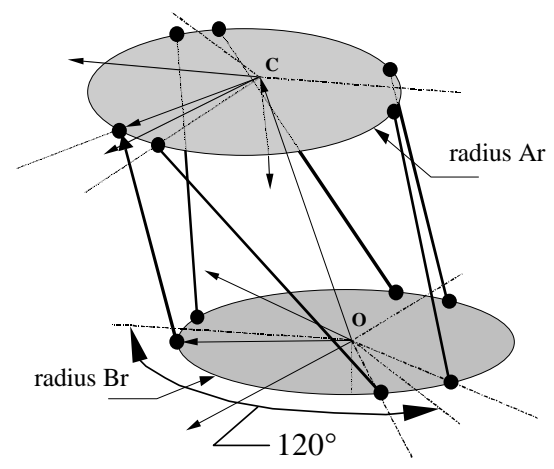

Figure 7 - Kinematic representation of the typical circular layout Stewart Platform (hexapod) mechanism

It is feasible to change the layout of the hexapod (thereby deviating from the standard circular geometric arrangement of the attachment points) in order to achieve a greater and better-distributed workspace, is also possible ${ }^{1,3}$. For example, one can theoretically place the hinge-points (gimbals) anywhere in space such as on two concentric, noncoplanar circles $^{2}$, rather than on one circle (Figure 8).

It is also easy for some manufacturers to change the "cut-length" of the actuator cylinders and piston rods,

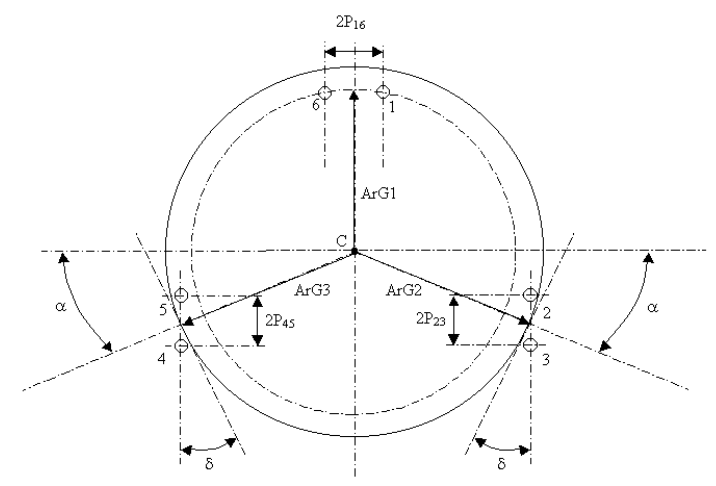

Figure 8 - Layout of double-concentric platform

while maintaining the original design of the hinge forks, manifolds, cushioning buffers and end blocks. The actuator stroke length may also be specified by the design requirements (rather than arbitrarily chosen multiples of prime numbers, as is often the case).

However, the designer must also prevent the platform from ever approaching singular conditions. In this situation, the ratio of actuator displacements to the resulting platform displacements is very low, meaning that the positioning accuracy may suffer, the mechanical loads can become high, or the control of the system difficult to achieve.

By using an optimization program, all of the above "free design variables" and constraints can be combined to yield the most suitable architecture tailored to the specified motion envelope.

A method of optimizing the motion-base ${ }^{1,3}$, based on the following steps, was developed and tested. The original reference ${ }^{1}$ provides more details. The salient points are explained here:

1. Use the basic concept of the Stewart Platform.

2. Establish which geometric parameters should be allowed to vary and by how much, and which should remain fixed.

3. Fit the shape and size of these required motions (as developed earlier in this paper) into the available simulator motions by altering its geometry. This is accomplished through a mathematical optimization.

4. Check that the leg forces are within reasonable limits, that the legs do not exceed their minimum or maximum lengths, and that the legs do not make physical contact with each other.

5. Iterate when necessary.

To simplify the process of mathematical optimization, the six-dimensional trajectories were approximated with the six-dimensional hyper-ellipsoid circumscribing their. In step 3 above, these ellipses are inserted into the workspace of the mechanism, and this workspace "grown" to allow the larges possible ellipse to be just contained within its space ${ }^{1,2}$. Increasing the workspace and changing its relative shape is made possible by relocating the hinge points and, if necessary, changing the characteristics of the actuators, namely their cut lengths.

\section{Example: Integrated design of an optimum Wright Flyer simulator motion cueing system and motion- base mechanism}

\section{The aircraft model}

For this analysis, a linear model approximation of Wright Flyer dynamics has been used. Culick ${ }^{6}$ analyzed the flight characteristics of the Wright Flyer and confirmed that the aircraft was indeed longitudinally and laterally unstable. For the longitudinal mode, the short-period mode is unstable, while for the lateral mode it is the spiral mode that is heavily unstable. From the linear state-space model the transfer functions for pitch attitude and roll attitude control were derived, Eq. 4. Note that $\ddot{a}_{c}$ is the canard angle, while $w r$ represents the wing warp plus rudder interlink. 


$$
\begin{aligned}
& H \frac{\theta}{\delta_{c}}(s)=\frac{\left(15.0941 s^{2}+71.6512 s+32.3732\right)}{\left(s^{4}+9.113 s^{3}-8.261 s^{2}-1.2524 s-22.6527\right)} . \\
& \text { and }
\end{aligned} .
$$

The bode plots of these transfer functions are presented in Fig. 9.
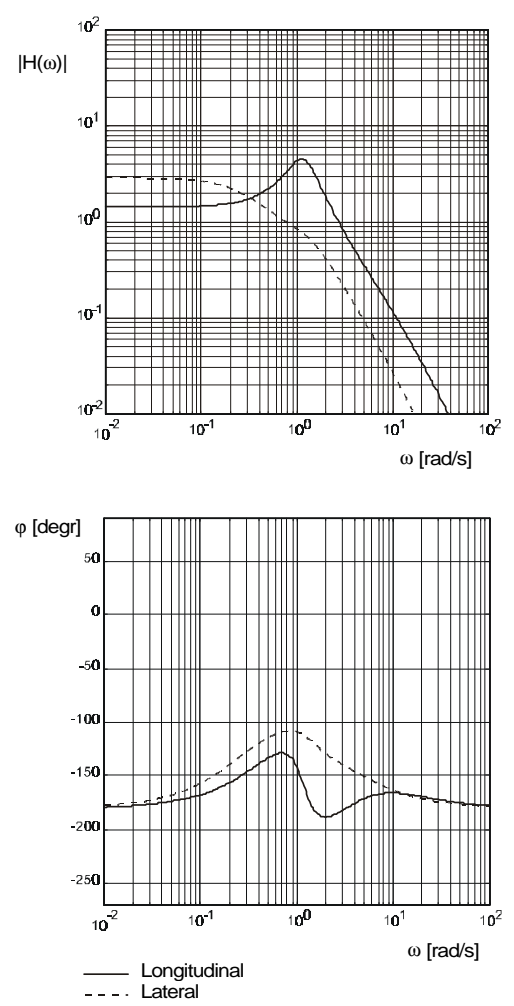

Figure 9 - Bode plot of the linear aircraft model

\section{Pilot model adaptation}

For the pilot model adaptation, the sensory weighting coefficients $\mathrm{W}_{\mathrm{i}}$ of Figure 2, must now be chosen. This is accomplished through an optimization procedure using the cost function of Eq. 1. The Bode plot of the adapted pilot model with and without motion feedback in the disturbance task is presented in Figure 10. Table 1 presents the model adjustment and closed-loop performance. Note that in the pilot model, a small time delay together with a secondorder system to describe the neuro-motor system dynamics replace the information processing time delay.

From Table 1, it is clear that during the disturbance task, the pilot model primarily uses vestibular feedback, and that the phase margin for pitch control is small. In the maneuver task, it is the visual rate feedback that serves as the primary variable. In the case of the Wright Flyer, however, there is no such clear preference (for either visual or for vestibular motion feedback) during the maneuver task.

Table 1 - Pilot model adjustment and loop closure results for longitudinal and lateral control, disturbance task and maneuver task, and for visual only, visual

\begin{tabular}{|c|c|c|c|c|c|c|}
\hline $\begin{array}{c}\text { Disturbance } \\
\text { task }\end{array}$ & $\mathbf{W}_{\mathbf{C}, \text { att }}$ & $\mathbf{W}_{\mathbf{C} \text {, rate }}$ & $\mathbf{W}_{\text {SCC }}$ & $\begin{array}{c}\text { STD } \\
\text { error } \\
{[\mathrm{deg}]}\end{array}$ & $\begin{array}{c}\omega_{\mathrm{c}} \\
{[\mathrm{rad} / \mathrm{s}} \\
]\end{array}$ & $\begin{array}{c}\boldsymbol{\varphi}_{\mathbf{m}} \\
{[\mathrm{deg}]}\end{array}$ \\
\hline \multicolumn{7}{|l|}{ Pitch } \\
\hline Visual only & 0.750 & 0.330 & 0 & 4.961 & 3.550 & 14.2 \\
\hline Visual and motion & 1.0491 & 0.0232 & 0.4931 & 3.100 & 3.718 & 40.67 \\
\hline CW2 washout & 0.8500 & 0 & 0.8997 & 4.695 & 4.294 & 15.35 \\
\hline Opt. washout & 0.8832 & 0 & 0.5485 & 4.332 & 3.475 & 29.27 \\
\hline \multicolumn{7}{|l|}{ Roll } \\
\hline Visual only & 1.3421 & 0.3952 & 0 & 1.478 & 1.366 & 65.07 \\
\hline Visual and motion & 1.8315 & 0 & 0.752 & 1.102 & 1.614 & 74.59 \\
\hline CW2 washout & 1.656 & 0 & 0.8973 & 1.265 & 1.388 & 74.04 \\
\hline Opt. washout & 1.7138 & 0 & 0.7717 & 1.196 & 1.430 & 71.22 \\
\hline $\begin{array}{c}\text { Maneuver } \\
\text { task }\end{array}$ & $\mathbf{W}_{\mathbf{C} \text {, att }}$ & $\mathbf{W}_{\mathbf{C}, \text { rate }}$ & $\mathbf{W}_{\text {SCC }}$ & $\begin{array}{c}\text { STD } \\
\text { error }\end{array}$ & $\omega_{\mathrm{c}}$ & $\varphi_{\mathrm{m}}$ \\
\hline Pitch & & & & [deg] & {$\left[\begin{array}{c}{[\mathrm{rad} / \mathrm{s}} \\
]\end{array}\right.$} & [deg] \\
\hline Visual only & 0.10 & 0.273 & 0 & 2.116 & 2.097 & 30.79 \\
\hline Visual and motion & 1.60 & 0.1727 & 0.6758 & 1.517 & 2.536 & 49.69 \\
\hline CW2 washout & 1.3903 & 0 & 1.1 & 1.886 & $\begin{array}{c}1.896 \\
8\end{array}$ & 55.65 \\
\hline Opt. washout & 1.55 & 0.04 & 0.80 & 1.609 & 2.373 & 40.61 \\
\hline \multicolumn{7}{|l|}{ Roll } \\
\hline Visual only & 1.4822 & 0.5283 & 0 & 1.242 & 1.617 & 62.41 \\
\hline Visual and motion & 5.2788 & 0 & 2.8085 & 1.160 & 1.366 & 63.55 \\
\hline CW2 washout & 2.3115 & 0.2897 & 1.3227 & 1.317 & $\begin{array}{c}1.105 \\
8\end{array}$ & 63.33 \\
\hline Opt. washout & 3.3349 & 0.1167 & 2.0299 & 1.279 & 1.185 & 61.55 \\
\hline
\end{tabular}
and motion (real flight) and simulated flight with optimal washout and the CW2 classical washout filter (Nahon and Reid, 1986).

\section{Washout algorithm parameter optimization}

After the pilot model has been adapted to the aircraft dynamics, the pilot model parameters are fixed, and the pilot simulated-aircraft loop is established, Fig. 10. The washout filter parameters are optimized for the simulation of the Wright Flyer with a lumped simulation time delay of 70 milliseconds present. Next, the parameters of the tilt-coordination filter and the high-pass rotation filter can be adapted using the cost function $\mathbf{J}$ of Eq. 1. In Fig. 11, as an example, the cost function is shown with respect to the tilt- coordination and rotation filter bandwidth $\omega_{n}(\mathrm{Lp})$ and $\omega_{\mathrm{n}}(\mathrm{Hp})$, respectively. In most cases, the cost function $\mathrm{J}$ can be described by a valley as a function of the break frequencies of the high-pass rotational filter, and the lowpass tilt coordination filter. After the washout parameters have been established, the pilot model may be adapted to 

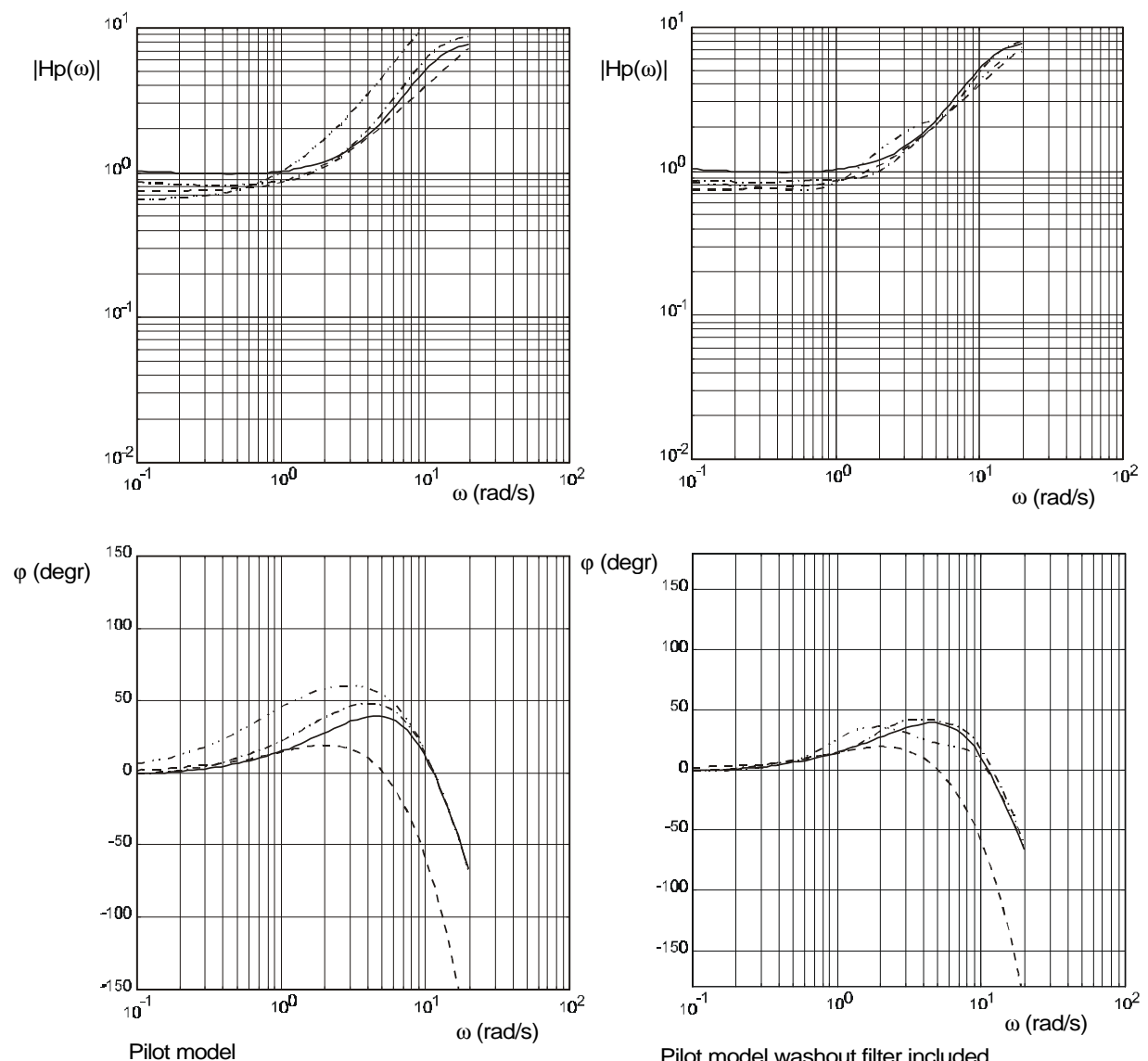

Pilot model washout filter included

$$
\begin{aligned}
& \text { - - - - - Visual feedback, real aircraft } \quad \text { Visual and vestibular feedback, real aircraft } \\
& \text { _....... } \quad \text { Visual and vestibular feedback, simulated aircraft, classical washout }
\end{aligned}
$$

Figure 10 - Bode plot of the pilot model for the Wright Flyer with and without motion feedback, and for the simulated aircraft with and without the influence of the washout incorporated.

the simulated aircraft and, subsequently, the pilot model adaptation to the real and the simulated aircraft compared. Fig. 10 presents the pilot model in the frequency domain for the real aircraft (i) without and (ii) with motion feedback, (iii) the optimized washout filters as described in this paper, and (iv) a classical washout filter ${ }^{15}$ as often used for transport aircraft training.

\section{Realizing the motion-base requirements}

As mentioned earlier, the motion-base mechanism can be designed to fit trajectories representing the desired simulated motions. When a mechanism is able to reproduce these trajectories with no further gain distortion, no saturation of its actuators, and no phase lags beyond those used in the pilot-model based analysis, then the pilot control strategy should remain the same in the simulator as in the aircraft.

Obtaining representative trajectory data, however, requires either flight recordings, or that the mathematical model of the aircraft be driven in the time domain. For obvious reasons, the latter was chosen

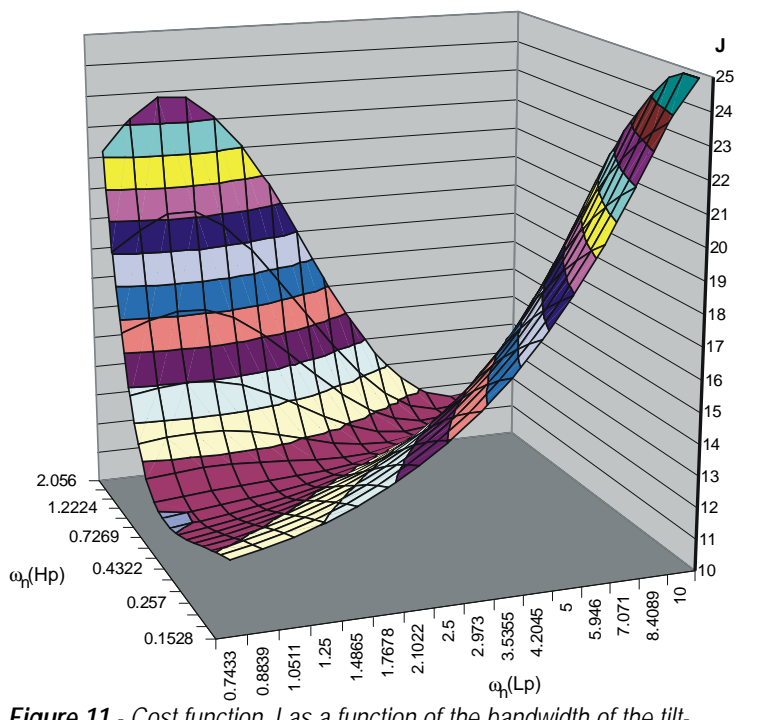

Figure 11 - Cost function $\mathrm{J}$ as a function of the bandwidth of the tiltcoordination $n(L p)$ and the rotation filter $n(H p)$ for the roll disturbance task. 
in this case, making use of a research flight simulator at the University of Liverpool which has a fullyintegrated model of the Wright Flyer coupled with flight controls and cueing devices.

The aircraft accelerations were registered during five representative maneuvers, and these later processed to generate the desired trajectories of the simulator. The positions and orientations, as well as their first and second derivatives, are thereby defined. The requirements of the motion-base - at least for the reproduction of these specific cases - are fully resident then in this trajectory data.

\section{Approximation of the trajectories}

The trajectories are then encapsulated into a reasonable mathematical approximation, represented by a six-dimensional "hyper-ellipsoid". If this ellipsoid contains these trajectories, and if it is possible to manufacture a motion system that is capable of generating a motion envelope at least as large as that ellipsoid, then it can be postulated that it is indeed possible to achieve our simulation needs.

Figure 12 shows the trajectories generated by the simulation of the 1903 Wright Flyer after they were passed through the optimized motion drive algorithm. In total, there are fifteen combinations possible (e.g. Surge-Heave, Surge-Sway, Heave-Sway, Pitch-Roll, Pitch-Sway, Pitch-Yaw, Pitch-Surge, etc.), though we have shown only four for clarity. Note that symmetry is applied to all lateral motions in order to allow the resulting simulator specification to reflect the symmetric capabilities of the aircraft (motions to the left or to the right should be possible with equality).

The hyper-ellipsoid has also been generated for these motions, and can also be seen in Figure 12. The size of this ellipsoid is mathematically determined so that the lengths of the primary axes represent the projections of the trajectories along the direction of that axis. In other words, the hyper-ellipsoid indicates the relative weightings for each of the six degrees-offreedom. If the trajectories include a large amount of cross-coupling, then they will exceed the boundaries of the hyper-ellipsoid. This issue will be resolved in the next section on mechanism design.

Figure 12 also shows that the ellipsoid is not necessarily centred about the zero point in the $X$ and $\mathrm{Z}$ degrees-of-freedom. The maneuvers and resulting trajectories dictate this offset, and one may need to pre-position the simulator prior to a mission or maneuvers in order to allow its simulation without hitting the actuator travel limits, and without having to over-specify the size of the motion-base.
Table 2 provides the weighting factors of the motions in each degree-of-freedom, which were generated by the optimal washout algorithm and trajectory approximation by the hyper-ellipsoid. Note that the trajectories also provide knowledge of the minimum required velocity and accelerations of the motion-base.
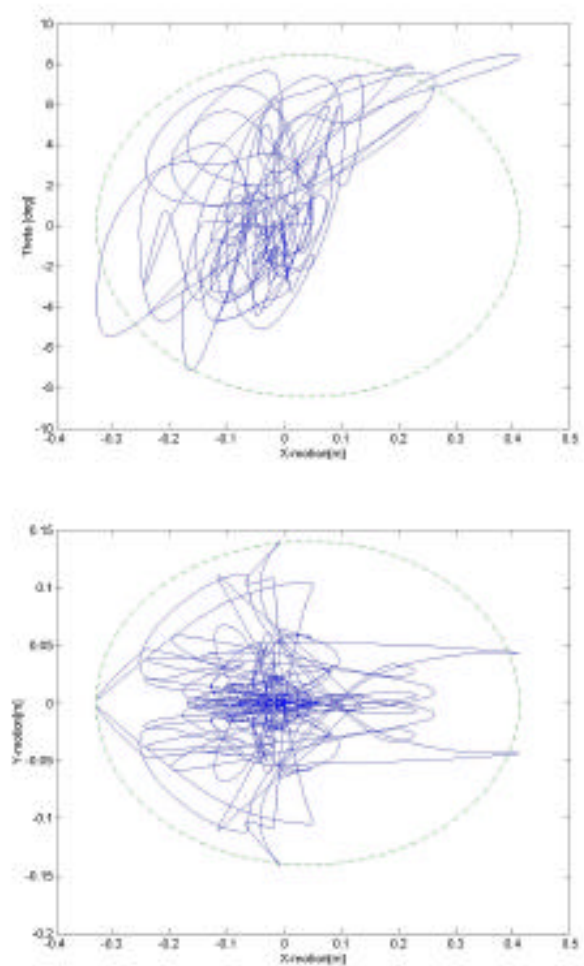

Figure 12 - Examples of the trajectories of the simulator motion system due to the flight files of the Wright Flyer and transformed by the optimal washout filter

Table 2 - Hyper-ellipsoid weighting factors generated by the analysis for the Wright Flyer example

\begin{tabular}{|c|c|c|}
\hline $\mathbf{X}$ & $\mathbf{Y}$ & $\mathbf{Z}$ \\
\hline $\pm 0.370 \mathrm{~m}$ & $\pm 0.140 \mathrm{~m}$ & $\pm 0.674 \mathrm{~m}$ \\
\hline Psi & Theta & Phi \\
\hline $\pm 4.2 \mathrm{deg}$ & $\pm 8.46 \mathrm{deg}$ & $\pm 8.47 \mathrm{deg}$ \\
\hline
\end{tabular}

\section{Motion-Base Design}

A tailored motion-base mechanism can now be designed to suit the requirements generated above.

The parameters of the hexapod, as described in Figure 8, were optimized in order to contain the resultant hyperellipsoid of Table 2. To demonstrate the effectiveness of the optimization, the geometric parameters of a very small commercially-available hexapod with actuators having a total stroke length of $20 \mathrm{~cm}$ were used. The workspace of 
the system was re-shaped to accommodate the requirement, in this case by changing only the locations of the attachment points. The actuator stroke length was maintained at $20 \mathrm{~cm}$. Most importantly - although minor, the changes to the geometry ensure that the shape is tailored to the simulation need, based on the pilot model, the vehicle dynamics, and the maneuvers that are flown.

The workspace and general layout of the final system is shown in Figure 13. Note that the vertical location of the lower joints is not co-planar, which allows more optimization freedom. It does mean though that the forward joints are located lower on the platform than the aft sets. The objective function increased from 0.332 to 0.430 , and the shape of the workspace became very conformant to that required, with little "wasted" capability. Note that these results show ONE possible solution; the actual design effort would yield several options, because of the presence of many local minima, each of which represents a specific improvement in the workspace, and having a specific geometry. The designer must then choose the most viable outcome based, for example, on manufacturing cost, and interface with the building or simulator structure.

Following this initial design step, a complete dynamics analysis is required during which the simulated mechanism is exercised throughout its envelope, and the loads in all members are checked. These verifications must include failure modes, such as run-away conditions, and valve- 0 conditions. While these analyses have been performed for systems optimized by the authors, their description exceeds the scope of this paper.

\section{Discussion and Conclusions}

The aforementioned process has demonstrated how one can specify the motion cueing algorithm and geometry of a flight simulator motion-base in an objective way. While the latest scientific knowledge in motion perception, and in motion-base design technologies have been applied here, the development of this analytical process is still in progress.

The general motion perception model discussed herein must be developed further before it can be fully applied to this type of analysis. Support for further research is
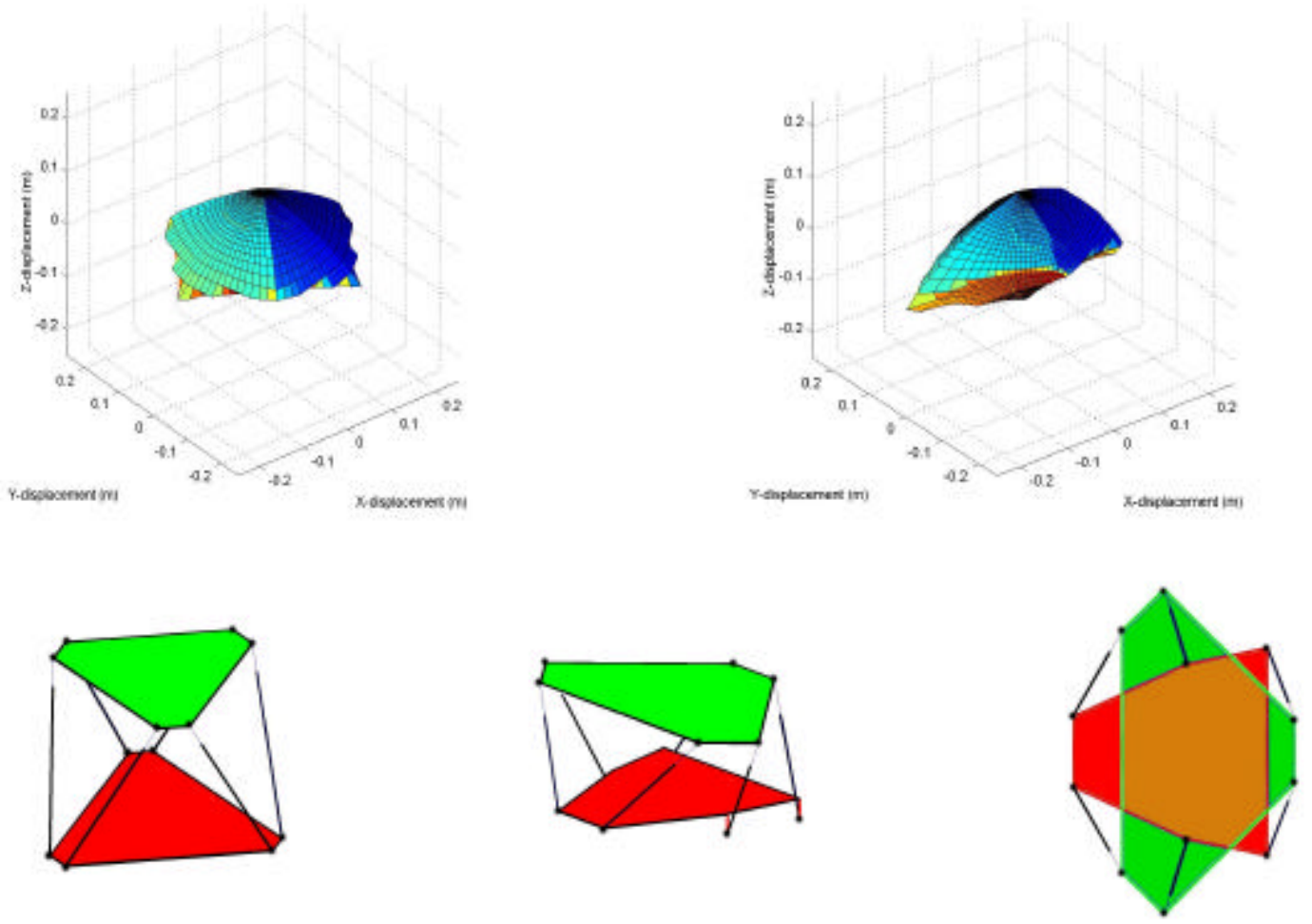

Conventional hexapod

Optimized hexapod

Figure 13 - Workspace (translations only shown) and mechanism geometry of a conventional (left) and optimized (right) motion cueing system for the Wright Flyer simulator. 
required in order to evaluate and fully validate the optimal washout filters as demonstrated in this paper. In addition, support is necessary to continue the development of motion perception models. Subsequently, with this perception model, the full use of the analysis will make it possible to determine the parameters of the high-pass linear motion filters as well.

The washout filter parameters obtained so far are indeed promising. By making a correct choice of the washout parameters, the pilot's control behaviour can be restored very closely to that in real flight.

By using a representative set of flight files, the motion system space required for the operation of the intended simulator can be obtained before the motion system is designed. A feedback of the results of the design into the analysis can be used to fine-tune the final washout algorithm and motion system.

The fact remains that this process allows the designer to achieve the most suitable washout characteristics and motion system performance, within the capabilities of existing mechanical hardware components. Cost-benefit studies can then be carried out to yield the "best" solution for the end-user customer.

The 1903 Wright Flyer was a highly-sensitive flight vehicle, and the capabilities of its developers and pilots are to be commended. Had Wilbur and Orville access to a motion-base simulator designed through techniques as those shown here, perhaps their design process could have been improved.

While aviation - and flight simulation - have come far since, motion cueing remains an area that needs attention.

\section{Acknowledgements}

Professor Gareth Padfield and Dr. Ben Lawrence of the University of Liverpool are thanked for the provision of Wright Flyer data. Bosch-Rexroth graciously provided motion system data.

\section{References}

1. Advani, S.K., "The Kinematic Design of Flight Simulator Motion-Bases". Ph.D. Thesis, Delft University of Technology. Delft University Press, 1998. ISBN 90-407-1672-2.

2. Advani, S.K., Giovannetti, D., Blum, M., "Design of a Hexapod Motion Cueing System for the NASA Ames Vertical Motion Simulator". In proceedings of AIAA Modeling \& Simulation Technologies Conference, Monterey, CA. August 2002. AIAA-2002-4794 CP.

3. Advani, S.K., Nahon, M., Haeck, N., "Optimisation of Six-Degrees-of-Freedom Flight Simulator Motion
Systems". AIAA J. of Aircraft, Vol. 36, No. 5, Sept-Oct. 1999.

4. Advani, S.K., and R.J.A.W. Hosman, "Integrated Motion Cueing Algorithm and Motion-Base Design for Flight Simulation". Proceeding of the conference on: Flight Simulation - The Next Decade. Royal Aeronautical Society, London, 10-12 May, 2000.

5. Bos, J.E., Bles, W. and Hosman, R.J.A.W., "Modeling Human Spatial orientation and Motion Perception". AIAA Modeling and Simulation Technologies Conference. Montreal, August 6-9, 2001. AIAA 2001-4248.

6. Culick, F.E.C., "What the Wright Brothers Did and Did Not Understand about Flight Mechanics - In Modern Terms". 37th AIAA/ASME/SAE/ASEE Joint Propulsion Conference, AIAA Paper No. 2001-3385, July, 2001.

7. Hess, R.A., "A Model for the Human's Use of Motion Cues in Vehicular Control". Journal of Guidance, Control and Dynamics. Vol. 13, No. 2, 1990, pp 476-482.

8. Hosman, R.J.A.W., "Pilots Perception and Control of Aircraft Motion". Ph.D. Thesis, Delft University of Technology, November 1996. ISBN 90-407-1384-7.

9. Hosman, R.J.A.W., "Are criteria for motion cues and time delays possible?" AIAA Modeling and Simulation Technologies Conference. Portland, Or. August 9-11, 1999. AIAA CP-99-4028.

10. Hosman, R.J. A.W. and Stassen, H.G., "Pilot's perception in the control of aircraft motions". Control Engineering Practices. Vol. 7, No. 11, pp. 1421-1428, 1999.

11. Hosman, R.J.A.W., Advani, S.K. and Haeck, N., "Integrated Design of Flight Simulator Motion Cueing Systems". Proceeding of the conference on flight Simulation. Royal Aeronautical Society, London, May, 2002.

12. Howard, F., "Wilbur and Orville - A Biography of the Wright Brothers". Alfred A. Knopf, New York, 1987. ISBN 0-394-54269-X.

13. McRuer, D.T., and H.R. Jex, "A Review of Quasi-Linear Pilot Models". IEEE Transactions on Human Factors in Electronics. Vol. HFE-8, no 3, September 1967.

14. Rasmussen, J., "Skills, Rules, and Knowledge; Signals, Signs, and Symbols, and Other Distinctions in Human Performance Models". IEEE Transactions on Systems, Man and Cybernetics, Vol. SMC-13, No. 3, May/June 1983.

15. Reid, L.D. and Nahon, M.A., "Flight Simulation MotionBase Drive Algorithms: Part 2 - Selecting the System Parameters". UTIAS (Univ. of Toronto) Report No. 307, CN ISSN 0082-5255. May 1986.

16. Stewart, D., "A Platform with six-degrees-of-freedom", in Proc. Inst. of Mechanical Engineers, vol. 180, part 1, no. 5, 1965-1966, pp. 371-386.

17. Teunissen, R., "The Future of Simulation and Training". Proceedings of the Royal Aeronautical Society Flight Simulation Conference "Can Flight Simulation Do Everything”. London, 19-20 May, 1999. 